\title{
The instruments used by the Italian centres for cognitive disorders and dementia to diagnose mild cognitive impairment (MCl)
}

\author{
Federica Limongi ${ }^{1} \cdot$ Marianna Noale ${ }^{1}$ (1) $\cdot$ Angelo Bianchetti ${ }^{2,3,4} \cdot$ Nicola Ferrara $^{3,4,5}$. Alessandro Padovani ${ }^{6}$. \\ Elio Scarpini ${ }^{7,8} \cdot$ Marco Trabucchi $^{3,4,9} \cdot$ Stefania Maggi ${ }^{1} \cdot$ for the MCI Working Group
}

Received: 8 June 2018 / Accepted: 27 August 2018 / Published online: 3 September 2018

(c) Springer Nature Switzerland AG 2018

\begin{abstract}
Aims The purpose of this study was to examine the tools used in Italy to diagnose mild cognitive impairment (MCI). Methods In collaboration with the Luigi Amaducci Research Consortium, the Italian Network of Alzheimer Evaluation Units prepared a questionnaire to describe how $\mathrm{MCI}$ is diagnosed in the Italian Centres for cognitive disorders and dementia (CCDD).

Results Most of the ninety-two CCDDs participating in the survey were located in hospitals (54.7\%); large percentages were coordinated by neurologists (50.8\%) and geriatricians (44.6\%). Almost all (98.5\%) used the Mini Mental State Examination to diagnose MCI; the Clock Drawing Test was also frequently used (83.9\%). Other neuropsychological, imaging and biomarker tests were utilized less frequently and a wide diversity in the instruments used was noted.

Conclusions According to the results, diagnoses of MCI are based on a multitude of instruments, with major differences in the clinical assessment of geriatricians and neurologists. Standardized testing protocols, validated instruments and cut-off points need to be identified and adopted by the CCDDs for assessing MCI.
\end{abstract}

Keywords Mild cognitive impairment · Diagnosis tools $\cdot$ Italian centres for cognitive disorders and dementia

Federica Limongi and Marianna Noale contributed equally to the study.

Marianna Noale

marianna.noale@in.cnr.it

1 CNR, Neuroscience Institute, Aging Branch, Via Giustiniani 2, 35128 Padua, Italy

2 Dipartimento di Medicina e Riabilitazione, Istituto Clinico S.Anna-Gruppo San Donato, Brescia, Italy

3 Società Italiana di Geriatria e Gerontologia (SIGG), Florence, Italy

4 Associazione Italiana di Psicogeriatria (AIP), Brescia, Italy

5 Dipartimento di Scienze Mediche Traslazionali, Università degli Studi di Napoli "Federico II", Naples, Italy

6 Neurology Unit, Dipartimento Scienze Cliniche e Sperimentali, Università degli Studi di Brescia, Brescia, Italy

7 Fondazione IRCCS Ca' Granda Ospedale Maggiore Policlinico, Milan, Italy

8 Università di Milano, Centro Dino Ferrari, Milan, Italy

9 University of "Tor Vergata", Rome, Italy

\section{Introduction}

Mild cognitive impairment (MCI) is an intermediate stage of cognitive impairment that often, but not always, represents a prodromal phase of dementia. In accordance with the international criteria based on the Key Symposium held in 2003, $\mathrm{MCI}$ is considered a clinical syndrome that has different aetiologies and clinical profiles including impairment not only in memory but also in other cognitive domains [1, 2]. New criteria for dementia recently published by the American Psychiatric Association in the fifth edition of the diagnostic and statistical manual for mental disorders (DSM-5) identified a pre-dementia stage of cognitive impairment defined as mild neurocognitive disorder (NCD). Sharing many features with MCI, the condition represents an initial phase of cognitive impairment that precedes the major neurocognitive disorder that corresponds to outright dementia [3].

Objective cognitive impairment, defined as poor performance on one or more neuropsychological tests or batteries, can refer to any cognitive domain such as executive functions, attention, language, memory and visuospatial skills. Depending on the type and number of cognitive domains 
found to be impaired, MCI has been classified into four subtypes: amnesic MCI (isolated memory deficit); multidomain amnesic MCI (difficulty of memory accompanied by a deficit in at least one other cognitive function, such as language, executive functions, visuo-spatial abilities); single domain non-amnesic MCI (cognitive decline involves a single domain not linked to memory, such as an isolated deficit in visuo-spatial functions); multidomain non-amnesic MCI (cognitive decline is linked to at least two cognitive functions, with the exclusion of memory) [1].

According to a recent review [4], MCI prevalence rates range between 16 and $20 \%$ in the general population. The incidence rate of dementia in patients with MCI is much higher than that observed in MCI-free individuals. The rate of progression to dementia in MCI patients varies from 20 to $40 \%$ [5]. However, individuals with MCI do not necessarily progress to dementia, and several studies have shown that they can remain cognitively stable or even recover normal cognitive status [6]. The high variability in prevalence rates and in the rate of conversion to dementia are undoubtedly linked to differences in the populations studied, in the duration of study's follow-up and, above all, in the diagnostic criteria utilized, as well as to the number and types of neuropsychological tests employed [7]. Currently, there is no consensus for agreed guidelines specifying the neuropsychological tests or batteries, the cut-off points and the number of tests or subtests that should be used to define impairment in a cognitive domain. Although many studies use the same or similar diagnostic criteria, the manner in which they are utilized is not necessarily the same $[2,8]$. The most frequently proposed screening tests include the Montreal Cognitive Assessment (MoCA [9]), the Mini-Mental State Exam (MMSE [10]) and the Mini-cog test [11, 12]. The MoCA is a screening tool that was developed specifically to detect MCI. Thirty items assess multiple cognitive domains including short-term memory, visuospatial abilities, executive functioning, attention, concentration, and working memory, language and orientation in time and place. The MMSE is a brief screening tool that is useful in estimating the severity of cognitive impairment and in assessing cognitive changes over time. The MMSE provides a measure of individual's orientation in time and place, immediate recall, short-term verbal memory, calculation, language, and construct ability. With respect to the MMSE, some researchers consider the MoCA more sensitive in differentiating MCI from normal cognition and dementia; it has been validated in diversified setting [13]. The Mini-Cog test only takes $3 \mathrm{~min}$, and is slightly influenced by education level. Mini-Cog is mainly used in screening patients with dementia and little research has been undertaken on whether it performs better than MMSE for screening MCI patients [14].

Cross-national comparative studies examining the prevalence and incidence rates of $\mathrm{MCI}$ and its progression to dementia provide important data for public health decisions and interventions for clinical research, but the lack of standardized instruments for its assessment often do not allow such studies.

Although the CRONOS project first [15] and the "Italian Dementia National Plan" of the Ministry of Health [16] later, have highlighted the challenge in Italy of formulating appropriate health care policies and of creating an integrated and coordinated care network for dementia, there are no specific indications on how to diagnose and manage MCI.

The current study aimed to evaluate the tools used in Italy by the Centres for Cognitive Disorders and Dementia (CCDD), formerly called Alzheimer Evaluation Units (AEUs), to diagnose MCI.

\section{Methods}

In collaboration with the Luigi Amaducci Research Consortium, the Italian Network of Alzheimer Evaluation Units (UNIVA) formulated a questionnaire (available upon request) to evaluate the tools used by the CCDDs to diagnose MCI. All the CCDDs located in the various regions of Italy were sent an e-mail with a description of the project and an invitation to participate. When a CCDD accepted the invitation, the credentials (username, password) needed to access the web form questionnaire which was to be completed between November 2017 and February 2018, were provided.

The questionnaire included three sections: the first collected information on the CCDD's characteristics (geographic location, a list of the individuals coordinating and manning the team, the days of the week the centre is open, the services it provides, the research activities it participates in). The second section regarded the centres' activity during the preceding month's time. It was dedicated to questions about the number of patients that were evaluated, including information regarding their ages and sex. There were also specific items concerning the patients who had been evaluated for the first time during that period and the diagnoses that were formulated. The third section focused on the tools used to formulate the diagnosis of MCI.

\section{Statistical analysis}

After the data quality control, categorical variables were summarized using percentages and frequency distributions and the continuous ones using median and interquartile range. The CCDDs groups were defined depending upon the type of coordination (neurologists vs geriatricians vs psychiatrists). The differences between the groups with regard to the patients' ages, the tests prescribed and the instruments used to formulate a MCI diagnosis were evaluated using the $\chi^{2}$ or Fisher's exact test for categorical variables and 
the Wilcoxon rank-sum test for quantitative ones. All the analyses were performed using SAS 9.4 software.

\section{Results}

Ninety-two CCDDs had access to the web form questionnaire; 70 completed the questionnaire.

More than half of the participating CCDDs $(n=35$; $54.7 \%)$ were set up in a hospital context, $12(18.8 \%)$ in University Clinics, 14 (21\%) in other types of health care services, and only $3(4.7 \%)$ in residential facilities for the elderly. Most of the centres were coordinated by neurologists $(n=33 ; 50.8 \%)$ or by geriatricians $(n=29 ; 44.6 \%)$; less than $5 \%(n=3)$ were coordinated by psychiatrists. The professional teams frequently included psychologists [present in 57 centers $(87.7 \%)$ ], neurologists [46 centers $(70.8 \%)$ ], nurses [41 centers (63.1\%)] and geriatricians [39 centers (60.0\%)]; psychiatrists, speech and rehabilitation therapists were present in less than $25 \%$ of the teams.

The total number of patients evaluated by the CCDDs participating in the survey during the preceding month was 7535; the median number of patients evaluated by each CCDD was $85(\mathrm{Q} 1=48, \mathrm{Q} 3=120)$. There were 4300 females who represented $60.3 \%$ of the patients, $552(8 \%)$ patients were younger than $65,1578(24.2 \%)$ fell into the 65-74 age groups, 3015 (43.7\%) fell into the $75-84$ age groups and $1750(24 \%)$ were 85 or older. An analysis of study results showed that the CCDDs coordinated by geriatricians evaluated a lower percentage of individuals who were 65 or younger (4\%) and a higher percentage of patients who were 85 or older (30\%), with respect to the CCDDs coordinated by neurologists (12\% and $18.5 \%$, respectively).

A total of 1916 individuals underwent a first evaluation over the previous month by the participating CCDDs; each CCDD evaluated a median of 21 persons $(\mathrm{Q} 1=10, \mathrm{Q} 3=38)$ for a first evaluation. The characteristics of the individuals evaluated for the first time by the CCDDs are outlined in Figs. 1, 2 and 3. One-fifth of the patients $(n=372)$ had a score of 26 or higher on the MMSE; 1151 patients (61.9\%) had a score that was lower than 24. A diagnosis of Alzheimer's disease was formulated for 509 patients (25.4\%), of vascular dementia for $216(11.6 \%)$ and of Alzheimer's plus vascular dementia for 380 patients (17.9\%). Overall, 1210 patients (65\%) were diagnosed with some type of dementia. Three hundred and twenty-two patients (17.5\%) who were being evaluated for the first time were diagnosed with MCI. A diagnosis was not formulated for approximately $10 \%$ of the patients undergoing evaluation by a CCDD for the first time.

The tests and exams most frequently prescribed during the first appointment were neuropsychological tests (for $n=1166$ patients; $82.4 \%$ ), brain computed tomography (CT scan) or brain magnetic resonance imaging (MRI) (for 938 $(52.5 \%)$ and $700(44.9 \%)$, respectively) and cerebrospinal fluid analysis (CSF) (for 240 patients; 15.8\%). Less than $10 \%$ of the individuals undergoing a first visit were prescribed genetic tests (apoE or others), amyloid positron emission tomography (PET) or single photon emission computed tomography (SPECT). CT scan was prescribed more often in the CCDDs coordinated by a geriatrician with respect to those coordinated by a neurologist ( $64.8 \%$ vs $40.8 \%$, respectively; $p=0.0175$ ). The CCDDs coordinated by neurologists
Fig. 1 The distribution of the Mini-Mental State Examination (MMSE) score among individuals evaluated by the CCDDs at the time of the first diagnosis

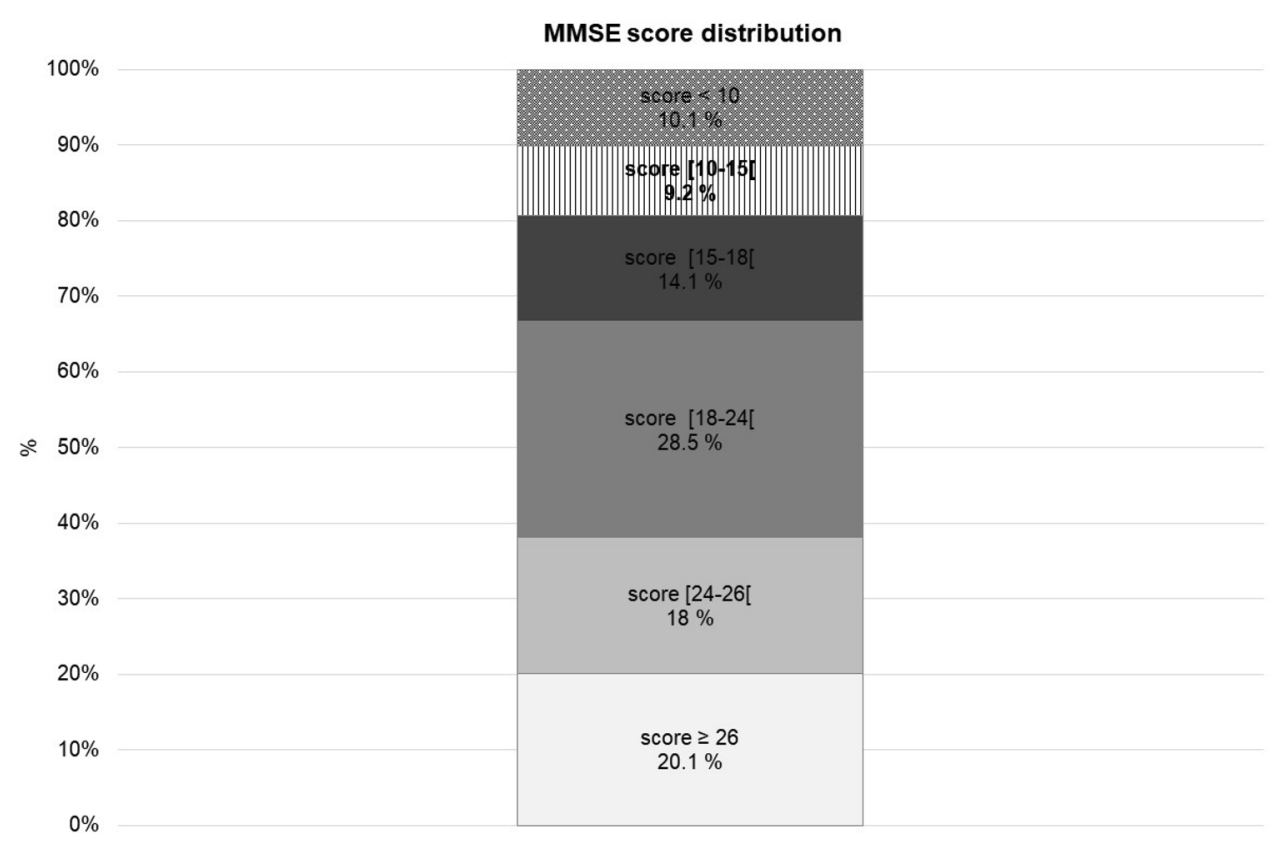


Fig. 2 Percentage of patients diagnosed with different disorders after the first visit to a CCDD
Fig. 3 Percentage of patients who were prescribed specific examinations (multiple answers allowed) after the first visit to a CCDD
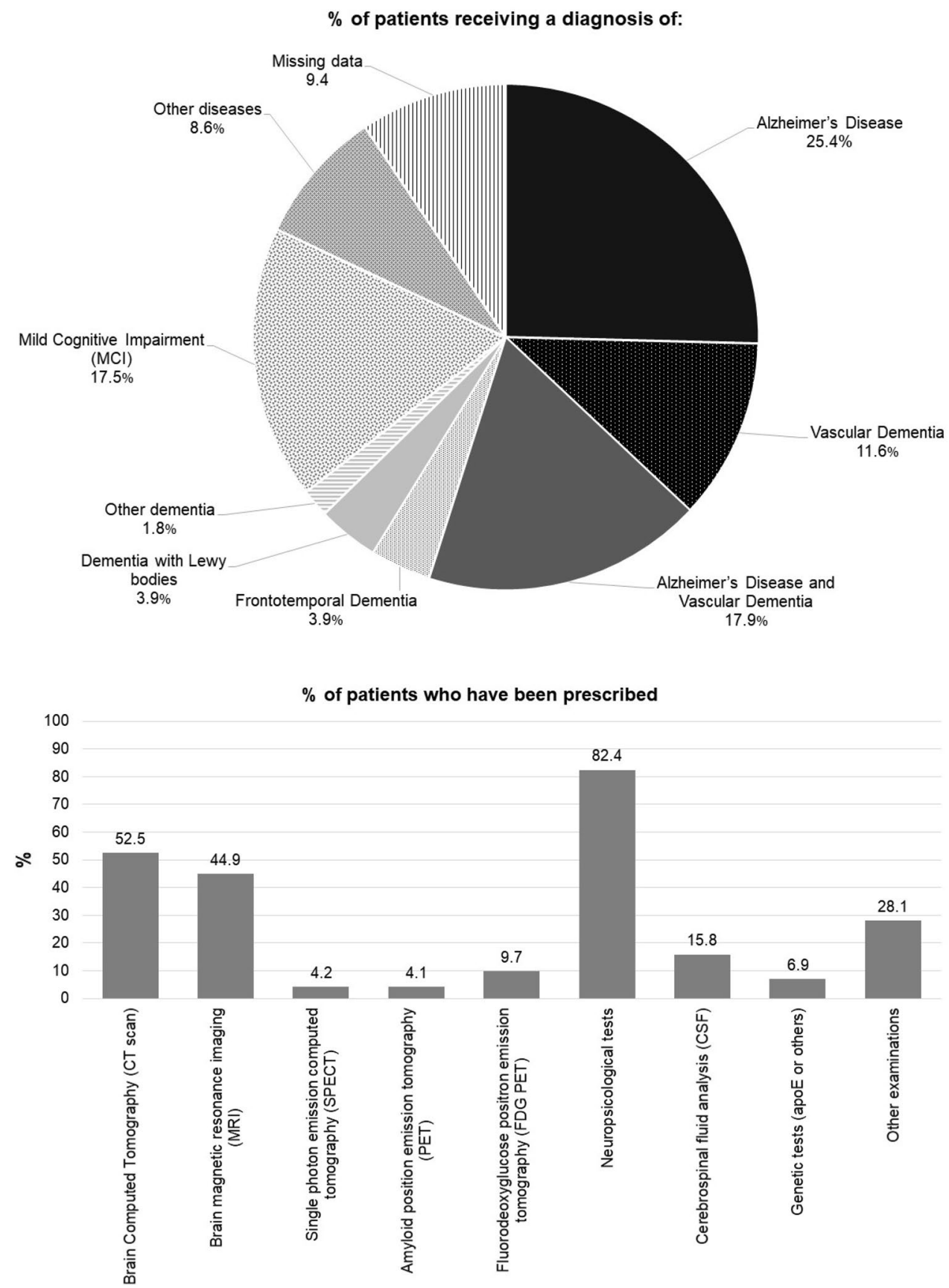

more frequently prescribed genetic tests $(11.4 \%$ vs $0.8 \%$; $p=0.0206)$ or CSF analysis ( $21.0 \%$ vs $11.0 \% ; p=0.0149)$.

Table 1 concerns the third section of the questionnaire which focused on the instruments used to reach a diagnosis of MCI. Almost all the centres (64/65 responded to this question) used the MMSE. The Clock Drawing Test [17] was used by $52 / 62$ centres (83.9\%). Less than $10 \%$ of the CCDDs (6/63) reported using the Alzheimer's Disease Assessment Scale (ADAS-cog; [18]). Eighty-five percent of the participating CCDDs (51/60 responded to this question) also evaluated depression but without specifying the instruments used; $80.7 \%$ (50/62) used the Neuropsychiatric Inventory (NPI; [19]). Sixty-nine percent of the CCDDs used other neuropsychological tests to formulate a diagnosis of MCI; the most frequently used were the MoCA (14/55 centres (25.5\%)), the Rey Auditory Verbal Learning Test (15/55 centres (27.3\%); [20]) and the Rey Complex Figure (15/55 centres (27.3\%); [21]).

The CCDDs coordinated by geriatricians less frequently reported using the Rey Complex Figure test, with respect to the CCDDs coordinated by neurologists $(12.5 \%$ and $41.4 \%$, respectively; $p=0.0202$ ). No other significant differences 
Table 1 A profile of the tools used to assess MCI at the first visit to a CCDD

\begin{tabular}{|c|c|c|c|}
\hline & $\begin{array}{l}\text { Number of centres that } \\
\text { answered this item }\end{array}$ & $\begin{array}{l}\text { Number of centres that acknowledge } \\
\text { using this instrument }\end{array}$ & $\%$ \\
\hline Mini-Mental State Examination (MMSE) & 65 & 64 & 98.5 \\
\hline Alzheimer's Disease Assessment Scale (ADAS-cog) & 63 & 6 & 9.5 \\
\hline Clock drawing test (CDT) & 62 & 52 & 83.9 \\
\hline Activities of daily living (ADL) & 58 & 62 & 93.6 \\
\hline Instrumental activities of daily living (IADL) & 59 & 63 & 93.7 \\
\hline Cumulative Illness Rating Scale (CIRS) & 58 & 26 & 44.8 \\
\hline Neuropsychiatric inventory (NPI) & 62 & 50 & 80.7 \\
\hline Depression or depressive symptomatology scales & 60 & 51 & 85.0 \\
\hline Scale caregiver stress assessment & 59 & 36 & 61.0 \\
\hline Other tests: & 55 & 38 & 69.2 \\
\hline Rey auditory verbal learning test & 55 & 14 & 27.3 \\
\hline Rey complex figure & 55 & 15 & 27.3 \\
\hline Montreal Cognitive Assessment (MoCA) & 55 & 14 & 25.5 \\
\hline Semantic and phonemic verbal fluency & 55 & 12 & 21.8 \\
\hline Trail making test & 55 & 12 & 21.8 \\
\hline Digit span & 55 & 10 & 18.2 \\
\hline Frontal assessment battery & 55 & 9 & 16.4 \\
\hline Visual search test & 55 & 7 & 12.7 \\
\hline Raven's progressive matrices & 55 & 7 & 12.7 \\
\hline Story recall test & 55 & 6 & 10.9 \\
\hline Token test & 55 & 5 & 9.1 \\
\hline Free and cued selected reminding & 55 & 5 & 9.1 \\
\hline Mental deterioration battery (MDB) & 55 & 4 & 7.3 \\
\hline Milan Overall Dementia Assessment (MODA) & 55 & 4 & 7.3 \\
\hline Stroop's test & 55 & 4 & 7.3 \\
\hline Apraxia test & 55 & 4 & 7.3 \\
\hline Corsi block-tapping task & 55 & 3 & 5.5 \\
\hline Babcock Story Recall Test & 55 & 3 & 5.5 \\
\hline Esame Neuropsicologico Breve & 55 & 2 & 3.6 \\
\hline Boston Naming Test & 55 & 2 & 3.6 \\
\hline Screening for Aphasia in Neurodegeneration & 55 & 1 & 1.8 \\
\hline Cinq mots test & 55 & 1 & 1.8 \\
\hline Three-objects-three-places test & 55 & 1 & 1.8 \\
\hline Dual task & 55 & 1 & 1.8 \\
\hline Tower of London test & 55 & 1 & 1.8 \\
\hline Visual object and space perception battery & 55 & 1 & 1.8 \\
\hline
\end{tabular}

emerged regarding the instruments to formulate a diagnosis of MCI and the CCDD coordination.

\section{Discussion}

The survey described here uncovered that there was high variability in the diagnostic investigation process and the test batteries used to evaluate specific cognitive domains in the CCDDs surveyed in Italy. Conversely, there was a high homogeneity with regard to the instruments used to evaluate the level of global cognitive function ( $99 \%$ of the practitioners declared that they used the MMSE), behavioural and psychological symptoms (81\% used NPI) and depression (85\% used specific instruments, although these were not specified).

According to the guidelines of the Italian Association of Psychogeriatrics (AIP) and the Dementia Study Group of the Italian Neurological Society (SINDEM), which are based on the diagnostic criteria proposed by the National Institute of Aging and the International Working Group, early detection of dementia can be achieved only if the cognitive tests 
and the tools utilized are capable of assessing behavioural and psychological symptoms. Since not only memory but also other cognitive functions need to be evaluated when individuals are being assessed for MCI [22], both rapid and global assessment tools such as the MMSE and tests investigating specific cognitive domains must be utilized, as they were by most of the CCDDs surveyed here.

Our questionnaire showed that psychiatrists play only a marginal role in the clinical work-up of CCDD team, thus arguing for behavioural and psychological symptoms being not extensively assessed during MCI assessment.

Another finding that emerged from the survey was the high percentage of patients who were diagnosed with dementia already during the first appointment (65\%). This would confirm the importance of setting up screening programs or protocols able to identify early stage cognitive decline and/or MCI-subtypes in the general population. Specific, targeted educational programs should train General Practitioners (GPs) and other health professionals to carry out cognitive screening in patients at early stages of the disease and to refer them to the nearest CCDDs that are located throughout the country. Until now the efficacy of cognitive impairment screening programs set up in primary care setting [23] has not been demonstrated, although screening tests performed by GPs seem to be useful if signs or symptoms of cognitive impairment are present [24]. It has been claimed that a therapeutic approach, based on pharmacological and non-pharmacological therapies (such as physical and cognitive stimulation) and monitoring cardiovascular risk factors, may be able to delay MCI progression and improve quality of life if it begun at an early stage of the disease [22, 25]. Timely MCI diagnoses could presumably lead to early interventions, as well as to integrated care pathways, better symptoms management, cost containment and delayed institutionalization [26].

The survey has some limitations, the major one being that not all the CCDDs responded to the questionnaire. Since questionnaires were anonymous, it was not possible to evaluate the difference between the centres that responded and those that did not. We can presume, however, that, on the basis of a self-selection principle, quality of the cognitive assessment was higher in the CCDDs participating in the survey, with respect to those that did not. The results cannot, therefore, be generalized to the overall Italian network. The fact that data collected did not allow us to evaluate if tests were used routinely or occasionally can be considered another limit.

In conclusion, the scientific community and the population at large are waiting the introduction of new pharmacological protocols that will be able to treat or slow down cognitive impairment progression. When this will become available, CCDDs will need to be better organized and to follow homogeneous diagnostic protocols and integrated care pathways. It is important that the numerous CCDDs located throughout Italy adopt standardized testing protocols, validated instruments and cut-off points to diagnose MCI and its subtypes. This would allow to obtain standardized rates of MCI and dementia and to reduce variability in reported rates of conversion from MCI to dementia. Moreover, in international comparative studies in the epidemiology of MCI and dementia.

Acknowledgements The "Survey on the instruments used by the Units Evaluating Alzheimer, now called Centres for Cognitive Disorders and Dementia, to reach a Mild Cognitive Impairment diagnosis" has been funded by an unrestricted grant by MSD ITALIA S.R.L.

The MCI Working Group:

Sara Antonucci (Chieti); Maria Grazia Arena (Messina); Stefano Avanzi (Mantova); Federica Barocco (Bergamo); Roberta Baschi (Palermo); Daniele Bellandi (Cremona); Carlo Adriano Biagini (Pistoia); Valentina Bono (Cuneo); Barbara Boselli (Vicenza); Gabriella Cacchiò (Ascoli Piceno); Emanuele Caggia (Ragusa); Veronica Caleri (Pistoia); Vincenzo Canonico (Napoli); Salvatore Caratozzolo (Brescia); Gabriele Carbone (Roma); Daniela Carta (Oristano); Maria Chiara Cavallini (Firenze); Giuliano Cerqua (Caserta); Alberto Cester (Venezia); Annalisa Chiari (Modena); Elisabetta Coppi (Monza e Brianza); Antonello Cossu (Oristano); Beatrice Cossu (Brescia); Maria Immacolata Cozzolino (Roma); Marina D’Agati (Palermo); Sanfilippo Daniele (Enna); Maria Letizia Del Re (Pescara); Babette Dijk (Genova); Andrea Fabbo (Modena); Sara Fascendini (Bergamo); Chiara Forlani (Mantova); Marino Formilan (Venezia); Carmine Fuschillo (Napoli); Michele Gennuso (Cremona); Carmela Gerace (Roma); Fabio Giacalone (Palermo); Barbagallo Giuseppe (Enna); Fabio Guerini (Brescia); Massimo Guido (Roma); Simona Linarello (Bologna); Fabio Lorico (Vicenza); Lucio Luchetti (Piacenza); Maurizio Luchetti (Perugia); Livia Ludovico (Parma); Mariacristina Lupinetti (Pescara); Savina Luvié (Piacenza); Michela Marcon (Vicenza); Donatella Mariani (Monza e Brianza); Giovanna Masone Iacobucci (Roma); Lucia Meligrana (Vicenza); Paola Melzi (Lecco); Vanda Menon (Modena); Giuliana Micale (Modena); Mauro G. Minervini (Bari); Roberto Monastero (Palermo); Enrico Mossello (Firenze); Francesco Nuccetelli (Chieti); Valentina Nucera (Parma); Anna Vittoria Marta Orsini (Napoli); Cristina Paci (Ascoli Piceno); Cristina Pagni (Pisa); Tommaso Piccoli (Palermo); Alessio Pietrella (Roma); Alberto Pilotto (Genova); Camilla Prete (Genova); Valeria Putzu (Cagliari); Michele Ragno (Ascoli Piceno); Monica Ricci (Roma); Roberto Rissotto (Genova); Alessandro Romorini (Milano); Mara Rosso (Cuneo); Luca Rozzini (Brescia); Maria Antonietta Rutigliani (Bari); Antonella Santillo (Napoli); Francesco Scoppa (Palermo); Piero Secreto (Torino); Barbara Senesi (Genova); Carlo Serrati (Genova); Andrea Spanò (Modena); Carla Stangalino (Milano); Antonio Tetto (Lecco); Alessandro Tiezzi (Arezzo); Gloria Tognoni (Pisa); Mirco Vanelli Coralli (Bologna); Concetto Vecchio (Messina); Daniela Viale (Cagliari); Giulia Vinceti (Modena); Marco Vista (Lucca); Elisa Zanacchi (Cremona); Patrizia Zannella (Torino).

Author contributions $\mathrm{AB}, \mathrm{NF}, \mathrm{AP}, \mathrm{ES}, \mathrm{MT}$ and SM planned the study and provided insights into analysis. FL and $\mathrm{MN}$ analysed the data and wrote the paper. All the authors critically reviewed the manuscript.

\section{Compliance with ethical standards}

Conflict of interest The sponsor was in no way involved in selecting the participants, reviewing the literature, analysing the data collected, drafting or reviewing the paper, or in the decision to submit the manuscript for publication. All the views expressed here are exclusively 
those of the authors. FL and MN declare that they have no conflict of interest to disclose. AB, MT, NF declare that they have no conflict of interest to disclose related to the paper. ES is member of the Eli Lilly and MSD International Scientific Board. SM declares grant from Takeda and Merck.

Statement of human and animal rights This article does not contain any studies with human participants performed by any of the authors.

Informed consent For this type of study formal consent is not required.

\section{References}

1. Winblad B, Palmer K, Kivipelto M et al (2004) Mild cognitive impairment-beyond controversies, towards a consensus: report of the International Working Group on Mild Cognitive Impairment. J Intern Med 256:240-246

2. Petersen RC, Caracciolo B, Brayne C et al (2014) Mild cognitive impairment: a concept in evolution. J Intern Med 275:214-228

3. American Psychiatric Association (2013) Diagnostic and statistical manual of mental disorders, 5th edn. American Psychiatric Association, Washington, DC

4. Roberts R, Knopman DS (2013) Classification and epidemiology of MCI. Clin Geriatr Med 29:753-772

5. Limongi F, Siviero P, Noale M et al (2017) Prevalence and conversion to dementia of Mild Cognitive Impairment in an elderly Italian population. Aging Clin Exp Res 29:361-370

6. Matthews FE, Stephan BC, McKeith IG et al (2008) Two-year progression from mild cognitive impairment to dementia: to what extent do different definitions agree? J Am Geriatr Soc 56:1424-1433

7. Ward A, Arrighi HM, Michels S et al (2012) Mild cognitive impairment: disparity of incidence and prevalence estimates. Alzheimers Dement 8:14-21

8. Díaz-Mardomingo M, del C, García-Herranz, Rodríguez-Fernández S R et al (2017) Problems in classifying mild cognitive impairment (MCI): one or multiple syndromes? Brain Sci 7:111

9. Nasreddine ZS, Phillips NA, Bedirian V et al (2005) The montreal cognitive assessment, MoCA: a brief screening tool for mild cognitive impairment. J Am Geriatr Soc 53:695-699

10. Folstein MF, Folstein SE, McHugh PR (1975) Mini-mental state. A practical method for grading the cognitive state of patients for the clinician. J Psychiatr Res 12:189-198

11. Borson S, Scanlan J, Brush M et al (2000) The mini-cog: a cognitive 'vital signs' measure for dementia screening in multi-lingual elderly. Int J Geriatr Psychiatry 15:1021-1027

12. Langa KM, Levine DA (2014) The diagnosis and management of mild cognitive impairment: a clinical review. JAMA 312:2551-2561
13. O'Caoimh R, Timmons S, Molloy DW (2016) Screening for Mild Cognitive Impairment: comparison of "MCI Specific" screening instruments. J Alzheimer's Dis 51:619-629

14. Fage BA, Chan CC, Gill SS et al (2015) Mini-Cog for the diagnosis of Alzheimer's disease dementia and other dementias within a community setting. Cochrane Database Syst Rev (2):CD010860

15. Bianchetti A, Padovani A, Trabucchi M (2003) Outcomes of Alzheimer's disease treatment: the Italian CRONOS project. Int $\mathbf{J}$ Geriatr Psychiatry 18:87-88

16. Di Fiandra T, Canevelli M, Di Pucchio A et al (2015) The Italian Dementia National Plan. Ann Ist Super Sanità 51:261-264

17. Sunderland T, Hill JL, Mellow AM et al (1989) Clock drawing in Alzheimer's disease. A novel measure of dementia severity. J Am Geriatr Soc 37:725-729

18. Rosen WG, Mohs RC, Davis KL (1984) A new rating scale for Alzheimer's disease. Am J Psychiatry 141:1356-1364

19. Cummings JL, Mega M, Gray K et al (1994) Neuropsychiatric Inventory: comprehensive assessment of psychopathology in dementia. Neurology 44:2308-2314

20. Estévez-González A, Kulisevsky J, Boltes A et al (2003) Rey verbal learning test is a useful tool for differential diagnosis in the preclinical phase of Alzheimer's disease: comparison with mild cognitive impairment and normal aging. Int J Geriatr Psychiatry 18:1021-1028

21. Osterrieth PA (1944) Le test de copie d'une figure complex: contribution a l'etude de la perception et de la memoire [The Complex Figure Test: Contribution to the study of perception and memory]. Arch Psychol 28:1021-1034

22. Padovani A, Musicco M, Caltagirone C et al (2015) Raccomandazioni dell'Associazione Italiana di Psicogeriatria (AIP) e della Società Italiana di Neurologia delle Demenze (SINDEM) sulla diagnosi precoce della Malattia di Alzheimer. Psicogeriatria, Supplemento 1:5-55

23. Moyer VA, U.S. Preventive Services Task Force Screening for cognitive impairment in older adults (2014) U.S. Preventive Services Task Force recommendation statement. Ann Intern Med 160:791-797

24. Cordell CB, Borson S, Boustani M et al, Medicare Detection of Cognitive Impairment Workgroup (2013) Alzheimer's Association recommendations for operationalizing the detection of cognitive impairment during the Medicare Annual Wellness Visit in a primary care setting. Alzheimers Dement 9:141-150

25. Vanacore N, Di Pucchio A, Lacorte E et al (2017) From mild cognitive impairment to dementia: what is the role of public health? Recenti Prog Med 108:211-215

26. Dubois B, Padovani A, Scheltens P et al (2016) Timely diagnosis for Alzheimer's disease: a literature review on benefits and challenges. J Alzheimers Dis 49:617-631 